\title{
Principaux facteurs de la germination de Heracleum sphondylium L : importance de l'oxygène
}

\author{
P Jauzein, A Mansour \\ ENSH, laboratoire de malherbologie, 4, rue Hardy, RP 914, 78009 Versaillis Cedex
}

(Reçu le 22 mars 1991; accepté le 25 novembre 1991)

\begin{abstract}
Résumé - Heracleum sphondylium est une ombellifère de friches qui ne supporte pas un travail du sol intensif. Cette sensibilité s'explique non seulement par la biologie d'hémicryptophyte mais aussi par les caractères germinatifs; la levée s'effectue de façon très homogène au début du printemps, aussi bien en surface qu'en profondeur, si bien qu'aucun stock semencier ne peut persister en terrain perturbé.

La régularité des germinations à la sortie de l'hiver est la conséquence d'une levée de dormance par le froid et l'humidité, conditions nécessaires à la réduction des inhibitions tégumentaires mais, surtout, à la maturation de l'embryon; aussi bien la levée de dormance primaire que le remplissage de l'embryon ne se déroulent qu'à une température inférieure à $10^{\circ} \mathrm{C}$. Cette exigence thermique s'explique simplement par un besoin en oxygène relativement élevé, satisfait à une température où la diffusion de ce gaz est facilitée : meilleure solubilisation, limitation des réactions de fixation.

Les enveloppes (téguments et substances de réserve) ont, ainsi, un rôle complexe de régulation de la quantité d'oxygène et de la qualité d'éléments nutritifs disponibles. Après une phase d'hypoxie empêchant la germination à contresaison, l'embryon dispose en fin d'hiver du flux idéal pour son développement, correspondant à une atmosphère environnante riche de $10-15 \%$ d'oxygène.
\end{abstract}

immaturité embryonnaire / oxygénation / Apiaceae

Summary - Principal factors in the germination of Heracleum sphondylium L : importance of oxygenation. Heracleum sphondylium is an umbellifer found on uncultivated soil which cannot tolerate intensive cultivation. This sensitivity may be explained not only by the biology of the hemicryptophyte, but also by its germination characteristics: the seedlings emerge nearly simultaneously at the beginning of spring, near the surface as well as from deeper levels, in such a way that no live seeds can persist if the soil has been disturbed.

The regularity of germination at the end of winter is the consequence of dormancy breaking by cold and moisture, necessary conditions for the reduction of tegumentary inhibition, but more especially for the maturation of the embryo. Thus dormancy breaking as well as embryo swelling do not take place unless the temperature is below $10^{\circ} \mathrm{C}$. This thermal requirement may be explained simply by the need for a relatively high level of dissolved oxygen around the seed, which may be satisfied at a temperature at which the diffusion of this gas is facilitated, making it more soluble and limiting its fixation reactions. The seed envelopes (seed coats and endosperm) thus play a complex role in regulating the availability quantity of oxygen and nutritive elements. After a phase of hypoxia preventing germination in the off-season, there is an ideal flow rate for the development of the embryo by the end of winter, ie is when the surrounding atmosphere reaches a partial pressure of 10 to $15 \%$ oxygen.

embryo immaturity / oxygenation / Apiaceae

\section{INTRODUCTION}

Parmi les plantes herbacées présentes dans les champs cultivés, rares sont celles qui régulent la périodicité des levées de plantules grâce à des phénomènes de dormance embryonnaire (Jauzein, 1986). En fait, chez la plupart des mauvaises herbes, les "dormances" ont pour origine diverses inhibitions provenant de toutes les structures qui entourent l'embryon : tissus de réserves, téguments, enveloppes florales disséminées avec le fruit.

Heracleum sphondylium $L$ est une ombellifère (Apiaceae) exclue, par sa biologie d'hémicryptophyte, des parcelles où le sol est travaillé; elle se cantonne aux prairies, aux talus herbeux et à des vergers mal entretenus où le rang non perturbé lui sert de refuge (Jauzein, comm pers). 
Ce modèle a été choisi pour la corrélation entre une situation marginale par rapport aux champs cultivés et l'existence d'une profonde dormance embryonnaire (Stokes, 1952a).

En effet, la semence d'Heracleum est, à l'image de celle des autres ombellifères, un akène albuminé contenant un embryon de petites dimensions (moins de $0,8 \mathrm{~mm}$ de long); une fois isolé, cet embryon ne germe pas et peut donc être considéré comme dormant. Diverses études ont montré l'immaturité physiologique d'un tel embryon; il nécessite, pour acquérir la capacité à germer, une période de maturation ne pouvant s'accomplir qu'à basse température (Stokes, 1952a, b). Ce phénomène a été vérifié chez diverses hémicryptophytes de la même famille (Felmion et Henrickson, 1949; Janiesch, 1971; Lovett Doust et Lovette Doust, 1982); il se traduit dans la nature par une levée strictement printanière de ces espèces (Roberts, 1979). II peut s'expliquer (Stokes, 1953a, b) par un problème d'alimentation de l'embryon et tout particulièrement par un besoin en acides aminés spécifiques. Mais la température est-elle vraiment le facteur limitant?

C'est pour répondre à cette question et expliquer le comportement au champ de cette espèce que nous nous proposons de rechercher l'importance de certains facteurs du milieu dans l'évolution de cette postmaturation originale en insistant sur l'élément qui nous paraît fondamental : l'oxygène.

\section{MATÉRIEL ET MÉTHODES}

Les semences d'Heracleum ont été récoltées en septembre 1986 dans des vergers abandonnés de Montigny-le-Bretonneux (Yvelines), ou sur des pieds cultivés en conditions naturelles à l'École nationale supérieure d'horticulture de Versailles. Elles ont été pour partie conservées au sec, en bocaux de verre, dans les conditions ambiantes du laboratoire (environ $20^{\circ} \mathrm{C}$ ), et pour partie placées en plusieurs couches séparées par des papiers filtre humidifiés, dans des boîtes de Petri, à $2^{\circ} \mathrm{C}$.

Pour les diverses expériences, des échantillons sont régulièrement prélevés et placés dans des enceintes climatisées, soit à l'obscurité, soit en présence de lumière avec une photopériode de $16 \mathrm{~h}$.

Avant stratification ou au début de chaque test, les semences ont été désinfectées à l'hypochlorite de calcium ( $15 \%$ pendant $30 \mathrm{mn}$ ). Les akènes, ou les graines débarrassées des téguments du fruit, sont déposés dans des boîtes de Petri sur du papier Whatman $n^{\circ} 5$ imbibé avec $5 \mathrm{ml}$ d'eau distillée; un morceau d'éponge permet d'éliminer le surplus d'eau libre, tout en maintenant une réserve suffisante. Deux boîtes contenant chacune 25 semences ont été utilisées pour chaque test. Les embryons, isolés sous une loupe binoculaire, sont installés par 20 dans un tube de $4 \mathrm{~cm}$ de diamètre sur un petit cavalier de papier Whatman $\mathrm{n}^{\circ} 3$; on verse dans le tube $25 \mathrm{ml}$ d'eau distillée. Dans certains cas, indiqués dans le texte, nous nous sommes contentés de couper la pointe de l'albumen au ras de la loge contenant l'embryon.

Chaque test dure un mois (sauf indication particulière), les relevés de germination étant effectués le plus régulièrement possible. La germination d'une semence entière est considérée comme accomplie dès que sa radicule a percé les téguments; nous admettons de même qu'un embryon a germé quand sa radicule commence nettement à s'allonger et à se courber vers le milieu humide.

Lors de la mise en germination sous atmosphère contrôlée, les semences et embryons sont placés dans des cellules de verre parcourues par un mélange gazeux à base d'air et d'azote; les différentes teneurs en oxygène s'obtiennent par un jeu de capillaires, selon la méthode de Côme et Tissaoui (1968).

La mesure de l'absorption d'oxygène par les semences a été réalisée avec un appareil de Warburg, 2 fioles de référence permettant de corriger les variations de volume dues aux fluctuations de la pression atmosphérique. Chaque fiole contient, en plus du matériel à tester placé sur papier filtre, une coupelle renfermant $0,3 \mathrm{ml}$ de potasse à $20 \%$ et $1 \mathrm{ml}$ d'eau dégazée apportée au démarrage de l'expérience. La lecture est effectuée toutes les heures.

Les embryons ont été cultivés sur milieu nutritif liquide remplaçant l'eau d'imbibition. Parmi les milieux testés, nous avons retenu celui de Monnier enrichi de saccharose à raison de $20 \mathrm{~g} / \mathrm{l}$ (Monnier, 1971). À ce milieu ont été ajoutés divers acides aminés : arginine à $0,16 \mathrm{~g} / \mathrm{l}$, glutamine à $0,25 \mathrm{~g} / \mathrm{l}$, proline à $0,1 \mathrm{~g} / \mathrm{l}$.

Nous avons vérifié la validité des résultats par une analyse de variance d'après le logiciel Modli (Kobilinski et al, 1988). Tous les résultats présentés ici sont globalement significatifs; à titre indicatif, nous avons mentionné sur certaines figures les intervalles de confiance au seuil de $5 \%$.

\section{RÉSULTATS}

\section{Les besoins thermiques}

À température constante, les akènes frais d'Heracleum ne germent qu'en dessous de $10^{\circ} \mathrm{C}$ (fig 1). Les mêmes résultats sont obtenus sur des semences plus âgées (jusqu'à 2 ans) conservées au sec dans le laboratoire. Cependant, il est possible de faire germer certains lots (les moins dormants) jusqu'à une capacité de $20 \%$ à des températures de $10-20^{\circ} \mathrm{C}$ à condi- 


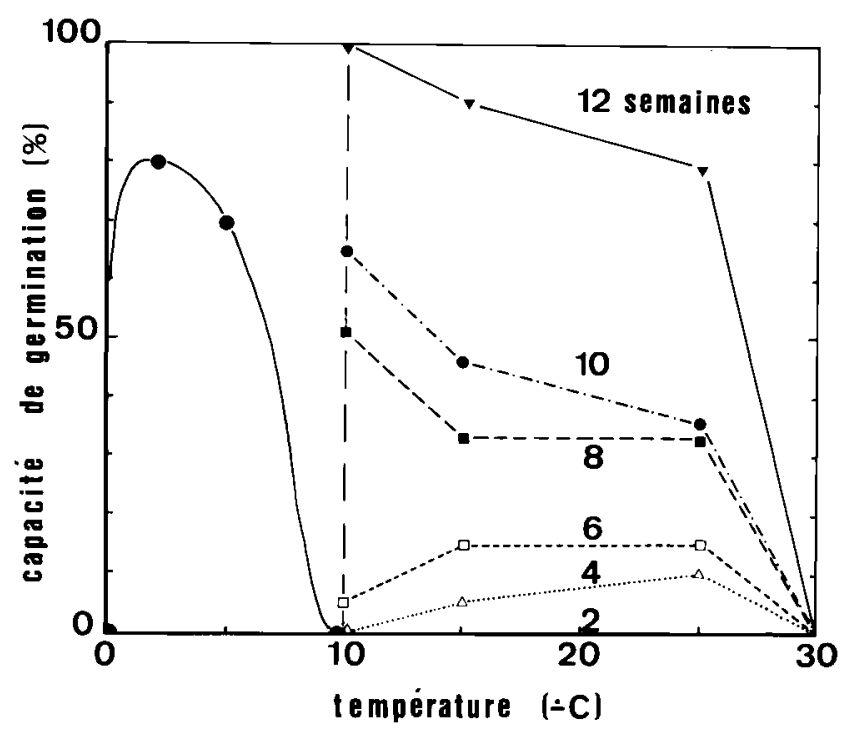

Fig 1. Influence de la température sur la germination à la lumière des semences d'Heracleum sphondylium; partie gauche (de 0 à $10^{\circ} \mathrm{C}$ ) à température constante pendant 4 mois; partie droite au cours d'une stratification $\left(2{ }^{\circ} \mathrm{C}\right.$ et $21 \%$ d'oxygène) dont la durée est indiquée (notation 1 mois après le changement de conditions).

tion de changer le papier filtre; nous reviendrons plus loin sur cette observation.

Au cours d'un prétraitement à $2{ }^{\circ} \mathrm{C}$ de semences imbibées, on observe une progressive levée de dormance (fig 1, partie droite). Pendant les premières semaines, l'optimum se situe de 15 à $25^{\circ} \mathrm{C}$, températures favorables à une bonne croissance. Par contre, sur akènes non dormants, obtenus après 11-12 semaines de prétraitement, les capacités de germination sont très élevées jusqu'à $10-15^{\circ} \mathrm{C}$, mais baissent à plus haute température pour s'annuler à $30^{\circ} \mathrm{C}$; c'est à $10^{\circ} \mathrm{C}$ que l'on obtient $100 \%$ de germination dans le délai le plus court (environ $8 \mathrm{j}$ ).

Par contre, quelles que soient la température de mise en germination et la durée du prétraitement à $2{ }^{\circ} \mathrm{C}$ d'embryons isolés ou d'embryons enveloppés d'un peu d'albumen, aucun de ces embryons ne germe (résultats non présentés); il s'agit donc bien d'une dormance embryonnaire.

Enfin, toutes ces expériences ont été répétées à la lumière et à l'obscurité; aucune différence significative n'apparaît, pas plus sur les capacités que sur les vitesses de germination : les semences d'Heracleum ne sont donc pas photosensibles.

Le comportement thermique mis ici en évidence correspond à celui de la plupart des mauvaises herbes tempérées (Jauzein, obs pers) : il peut s'expliquer par un besoin élevé d'oxygène pour la germination. Nous avons, pour le vérifier, placé les semences dans des atmosphères plus ou moins riches en oxygène.

\section{Les besoins en oxygène}

\section{Après un traitement à $2^{\circ} \mathrm{C}$ effectué dans l'air (fig 2A, B)}

Cette expérience met bien en évidence la progressive levée de dormance primaire à basse température. La durée optimale du traitement (donnant les meilleures capacités, obtenues en un temps minimal) correspond à 11 semaines de traitement; au-delà de 12 semaines, les akènes perdent progressivement leur viabilité (Mansour, 1990), en partie pour des raisons sanitaires.

Nous avons limité la figure 2 à la phase de levée de dormance. II faut au minimum 4 semaines de prétraitement pour que ces akènes ou certains embryons soient capables de germer.

Les akènes (fig 2B) ne germent pas en dessous de $10 \%$ d'oxygène. C'est l'air, avec $21 \%$ d'oxygène, qui permet la meilleure germination.

Pour les embryons, par contre (fig 2A), l'optimum correspond à une atmosphère contenant $15 \%$ d'oxygène; les concentrations plus fortes inhibent leur germination. À l'opposé (résultats non présentés), les embryons ne germent jamais en dessous de $1 \%$ d'oxygène et seuls quelques embryons non dormants ( $10 \%$ d'entre eux) réussissent à germer dans $1 \%$.

\section{Pendant la stratification}

La mise en évidence se fait à l'optimum d'oxygénation de $15 \%$ (fig 3 , A et B).

Pendant la levée de dormance à l'intérieur de l'akène, les meilleurs résultats sont obtenus dans l'air. On retrouve pratiquement le même histogramme dans les figures $3 \mathrm{~B}$ et $2 \mathrm{~B}$. Donc, aussi bien la phase de levée de dormance que la germination d'akènes non dormants nécessitent de fortes teneurs en oxygène; ce besoin explique le comportement thermique (fig 1).

Si l'embryon reste enveloppé d'un petit morceau d'albumen (fig $3 A$ ), les capacités germinatives restent fortement améliorées; ainsi, pendant la germination à $10^{\circ} \mathrm{C}$, les échanges continuent avec l'albumen qui fournit progressivement à l'embryon les éléments indispensables à son développement. C'est plus la qualité que la quantité qui semble primer à ces premiers stades. 

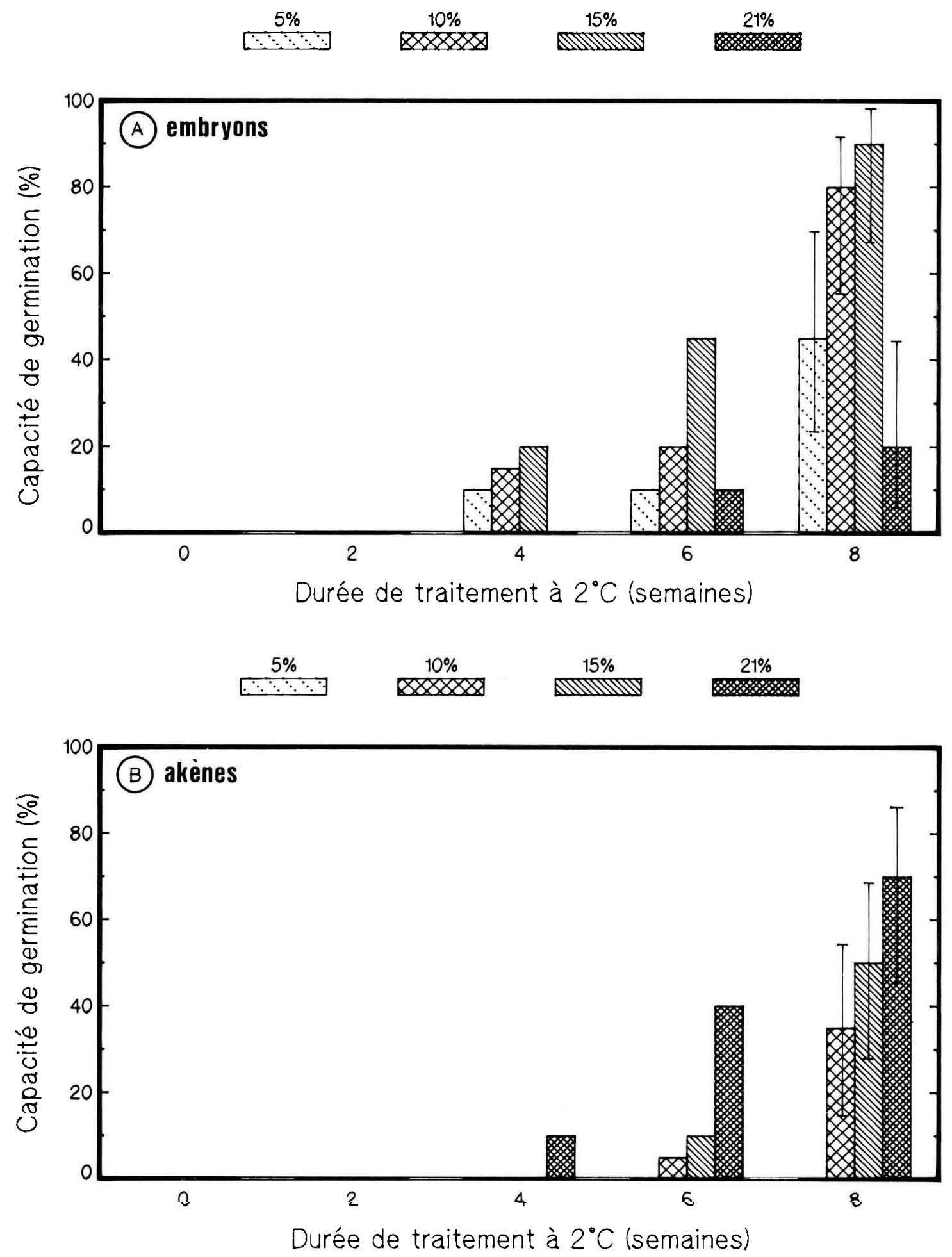

Fig 2. Influence de la teneur en oxygène sur la germination à $15^{\circ} \mathrm{C}$ et à la lumière d'embryons (A) et d'akènes (B) d'Heracleum sphondylium au cours d'un prétraitement des akènes $\left(2{ }^{\circ} \mathrm{C}\right.$ et $21 \%$ d'oxygène) : aucune germination entre 0 et $1 \%$ d'oxygène (sauf $10 \%$ d'embryons à $1 \%$ après 8 semaines de prétraitement).

Puisque l'optimum correspond, pour les semences entières, à une teneur de $21 \%$, alors que pour les embryons il correspond à $15 \%$, on peut penser que les enveloppes fixent la différence entre ces valeurs, plaçant ainsi les embryons dans une atmosphère interne favorable. 

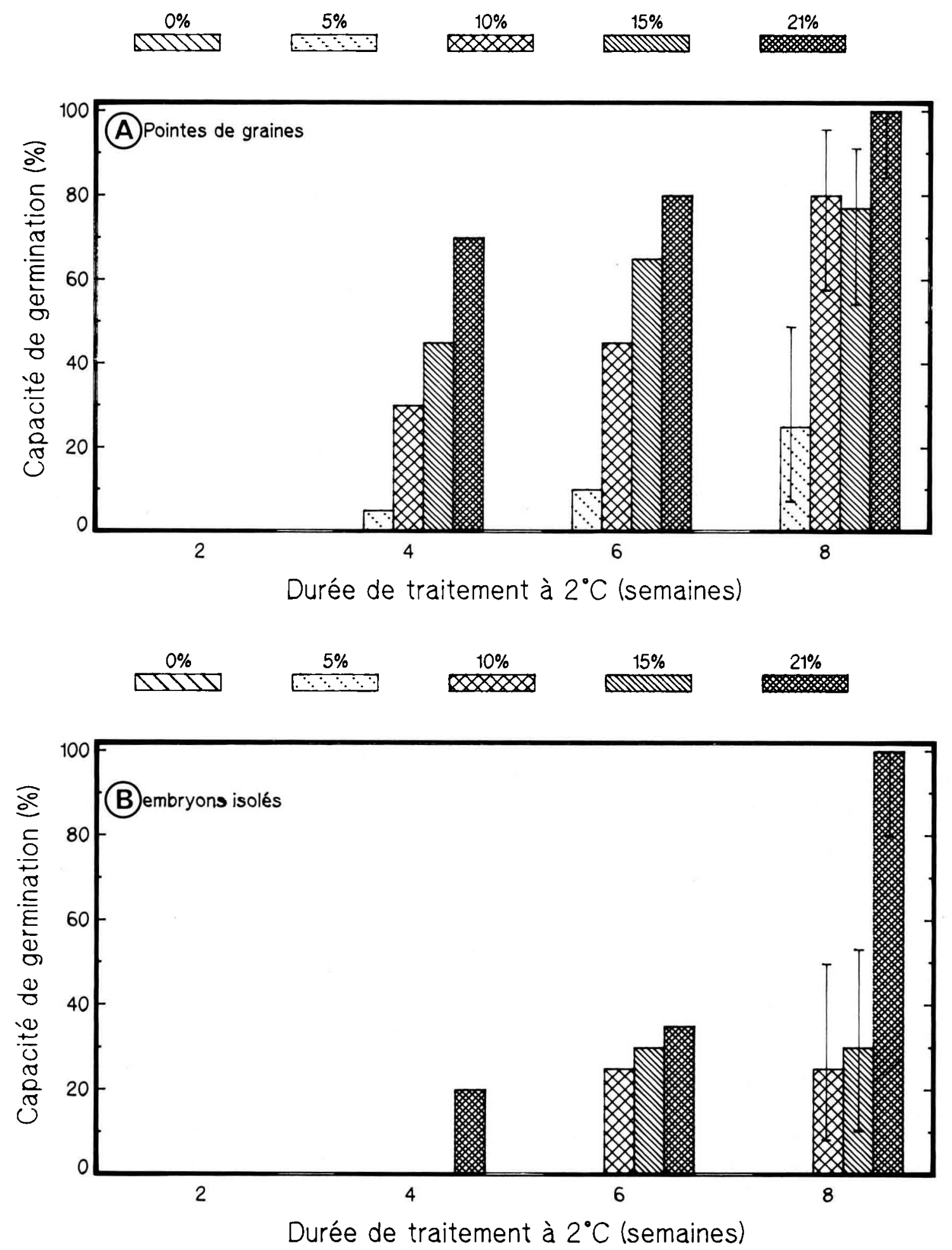

Fig 3. Influence de la teneur en oxygène sur l'efficacité d'un prétraitement d'akènes d'Heracleum sphondylium $\left(2^{\circ} \mathrm{C}\right.$ obscurité); la mise en évidence se fait par la germination en conditions optimales $\left(10^{\circ} \mathrm{C}\right.$, lumière, $15 \%$ d'oxygène $)$ d'embryons entourés d'un peu d'albumen (A) ou d'embryons isolés $(B)$.

\section{Rôle des enveloppes}

Divers degrés de décortication (fig 4) montrent bien l'effet inhibiteur des enveloppes. Sur la plupart des lots (surtout les récoltes précoces), le fait d'ôter les téguments du fruit améliore la germination pendant les premières semaines de stratification. Mais cette inhibition disparaît assez vite et, sur akènes non dormants, ne s'observe qu'à une température élevée (Man- 


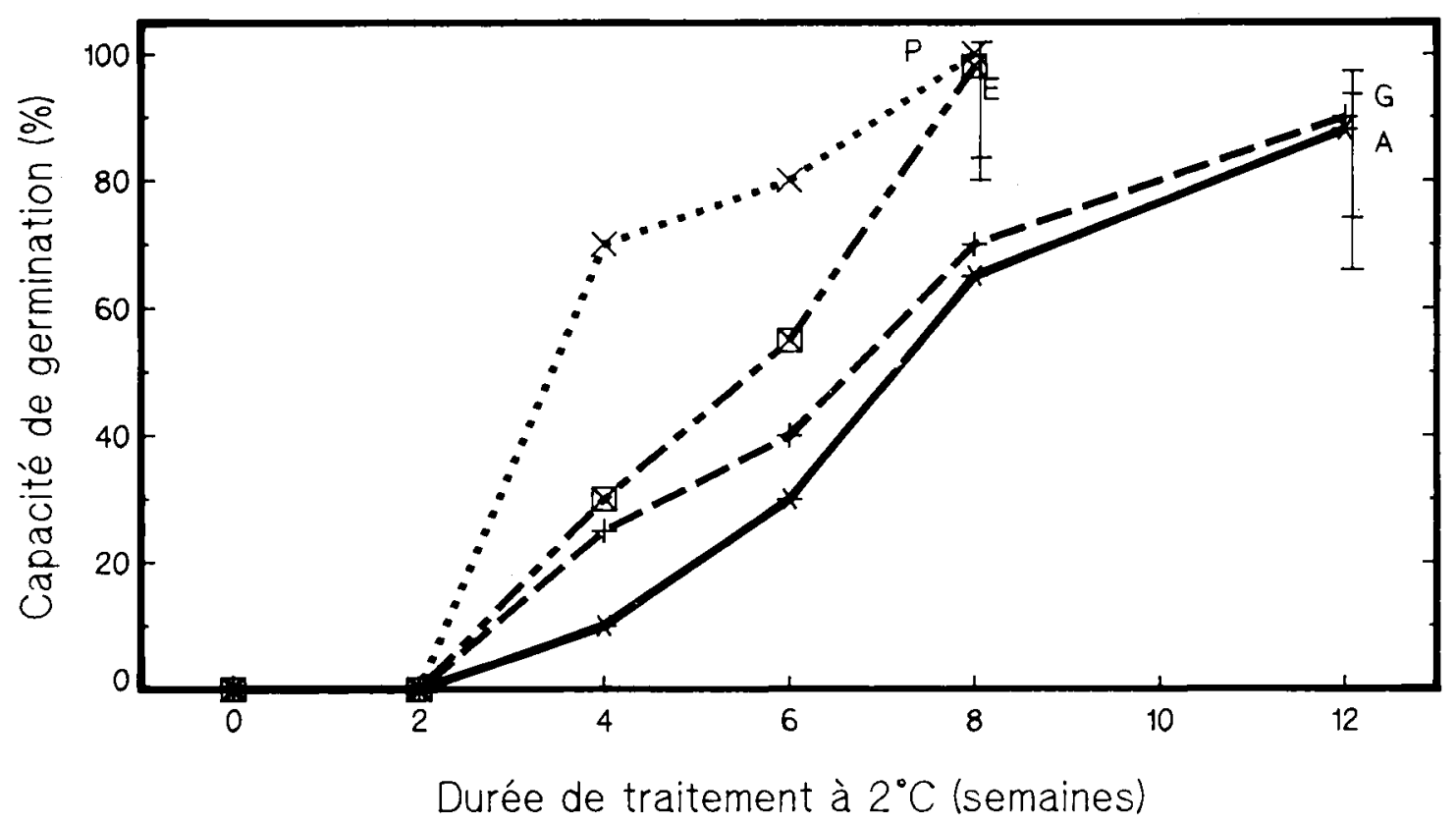

Fig 4. Influence du degré de décortication d'akènes d'Heracleum sphondylium sur l'évolution des capacités de germination (à $10^{\circ} \mathrm{C}$, lumière) au cours d'un prétraitement $\left(2^{\circ} \mathrm{C}\right.$, obscurité, $21 \%$ d'oxygène); les akènes (A) et les graines $(G)$ germent dans une atmosphère de $21 \% d^{\prime} \mathrm{O}_{2}$, les embryons (E) et les embryons enveloppés dans la pointe de l'albumen $(P)$ sont placés dans $15 \%$ d' $\mathrm{O}_{2}$.

sour, 1990); à 10 ou $15^{\circ} \mathrm{C}$ les différences ne sont pas significatives bien que les graines germent toujours un peu mieux; sur de nombreux essais, on constate une amélioration moyenne de $10 \%$ des germinations dans ces conditions. Cependant, l'inhibition tégumentaire reste très nette au niveau des vitesses de germination.

Le fait de supprimer une bonne partie de l'albumen permet de gagner environ 3 semaines; à la $8^{e}$ semaine de stratification, tous les embryons peuvent germer, alors que seulement $70 \%$ des graines germent. Il y a bien sûr les téguments des graines, mais ceux-ci sont très fins et réagissent sans doute comme les parois du fruit; l'inhibition plus persistante est plutôt à mettre au compte de réactions d'oxydation des substances de réserve de l'albumen. S'il ne reste qu'un petit bout d'albumen, l'effet inhibiteur est supprimé, et il ne subsiste que l'effet stimulant lié à l'alimentation de l'embryon.

II existe donc une double fixation d'oxygène, au niveau des téguments et au niveau de l'albumen. La fixation tégumentaire peut être mesurée par un appareil de Warburg (fig 5). Les akènes frais absorbent beaucoup plus d'oxygène que les graines; la différence correspond à la forte fixation au niveau des téguments du fruit. Par contre, après traitement au froid, la différence devient faible; l'inhibition disparaît pendant la levée de dormance embryonnaire. La différence entre graines dormantes ou non, constante bien que non significative, pourrait s'expliquer par la perte de capacité de fixation des fins téguments de la graine.

L'absorption des graines non dormantes représenterait ainsi la somme de la fixation au niveau de l'albumen et de la consommation de l'embryon.

Bien que l'inhibition tégumentaire soit souvent masquée par la forte dormance embryonnaire, et disparaisse rapidement lors d'un traitement au froid, elle n'en a pas moins un rôle important sur l'équilibre des gaz de la semence. Elle s'ajoute à la fixation de l'albumen qui présente ainsi 2 effets opposés; pour permettre la germination des embryons in vitro il faut donc supprimer les enveloppes et essayer de suppléer aux besoins métaboliques en les cultivant sur un milieu nutritif.

\section{Cultures sur milieu nutritif}

Des essais préliminaires de germination dans l'air ont donné des résultats décevants imputables à un excès d'oxygène; nous ne présenterons donc ici que les résultats obtenus dans une 


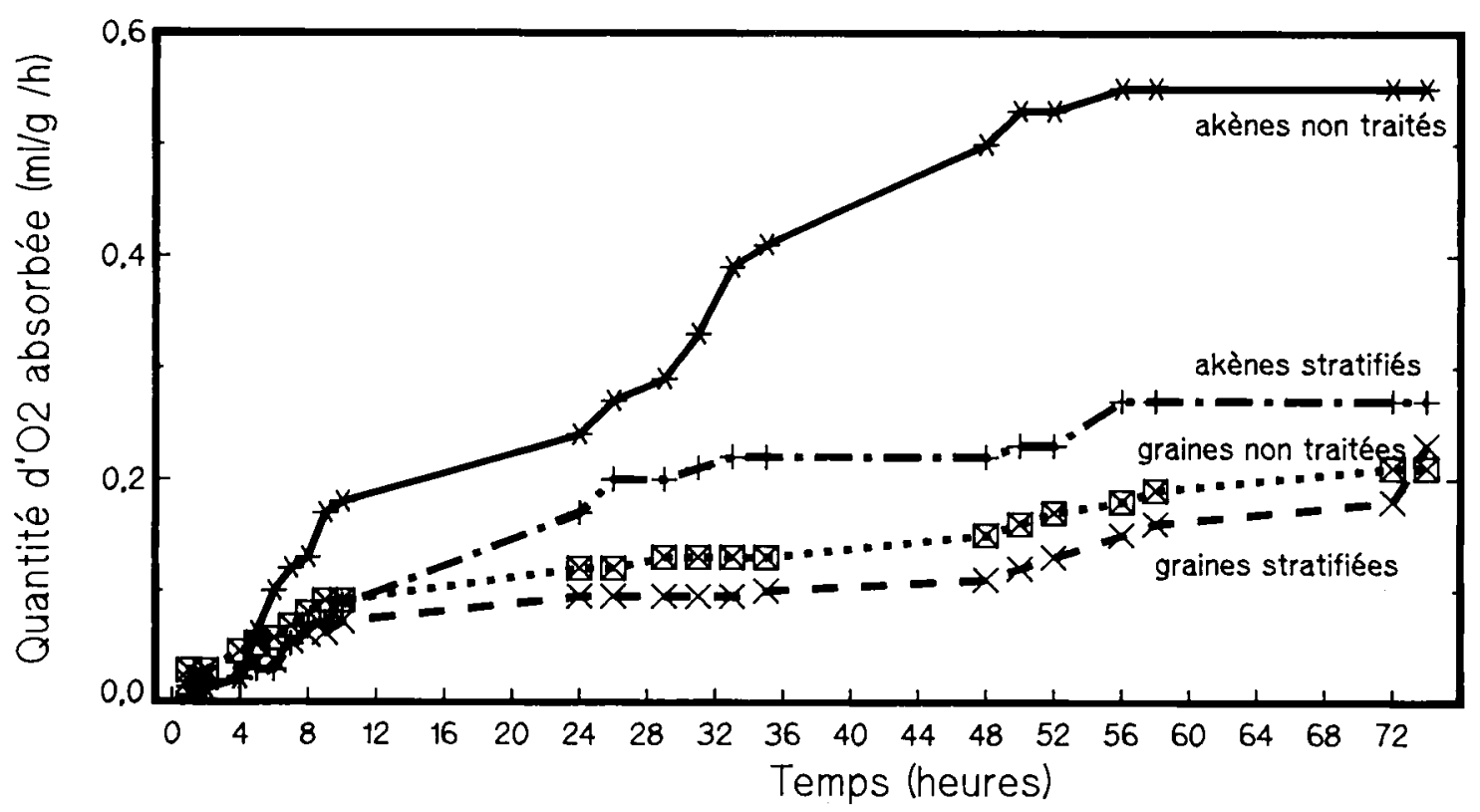

Fig 5. Absorption d'oxygène à $15^{\circ} \mathrm{C}$ de graines ou d'akènes d'Heracleum sphondylium dormants ou prétraités 11 semaines à $2{ }^{\circ} \mathrm{C}$ et $21 \%$ d'O$_{2}$.

atmosphère ne contenant que $15 \%$ d'oxygène (tableau I).

Sans prétraitement par le froid, il est possible d'obtenir la germination de la moitié des embryons avec l'apport de certains acides aminés (mélange arginine, glutamine et proline). Des milieux plus complexes devraient être testés pour améliorer ce score, sachant que certains éléments à faible dose peuvent suffire s'ils sont essentiels dans le métabolisme de l'embryon.

Après seulement 4 semaines de stratification d'akènes imbibés, il est possible d'obtenir la germination de tous les embryons en 8 j; le complément apporté par le milieu est donc amplement suffisant à ce stade. C'est pendant les premières semaines du prétraitement que la qualité du milieu fait défaut.

En comparant différents traitements améliorant les capacités et les vitesses de germination des embryons (fig 6), on voit que le milieu nutritif permet de diviser par 2 le besoin en froid.

\section{Induction d'une dormance secondaire}

Nous avons vu que les akènes placés dans des atmosphères à faibles pressions partielles en oxygène ne germaient pas (fig 2 et fig 7A). Si après un séjour en hypoxie on replace les akènes à l'air (fig 7B), les capacités de germina-

Tableau I. Capacités de germination (\%) en conditions optimales $\left(10^{\circ} \mathrm{C}\right.$, lumière, $15 \%$ d' $\left.\mathrm{O}_{2}\right)$ d'embryons d'Heracleum sphondylium placés sur milieu nutritif : milieu de Monnier enrichi de saccharose et de divers acides aminés (témoin sur eau distillée).

\begin{tabular}{|c|c|c|c|c|c|}
\hline Doses & Témoin & $\begin{array}{c}\text { Arginine } \\
\left(86.10^{-5} \mathrm{~mol}^{-1}\right)\end{array}$ & $\begin{array}{c}\text { Glutamine } \\
\left(17.10^{-5} \text { mol. } 1^{-1}\right)\end{array}$ & $\begin{array}{c}\text { L-proline } \\
\left(86.10^{-5} \mathrm{~mol}^{-1}\right)\end{array}$ & $\begin{array}{l}\text { Mélange } \\
\text { des } 3 \text { a a }\end{array}$ \\
\hline $\begin{array}{l}\text { Semences fraîches } \\
\text { notation à } 1 \text { mois }\end{array}$ & 0 & 10 & 30 & 55 & 50 \\
\hline $\begin{array}{l}\text { Semences stratifiées } \\
4 \text { semaines } \\
\text { notation à } 10 j\end{array}$ & 25 & non testé & non testé & 95 & 100 \\
\hline
\end{tabular}




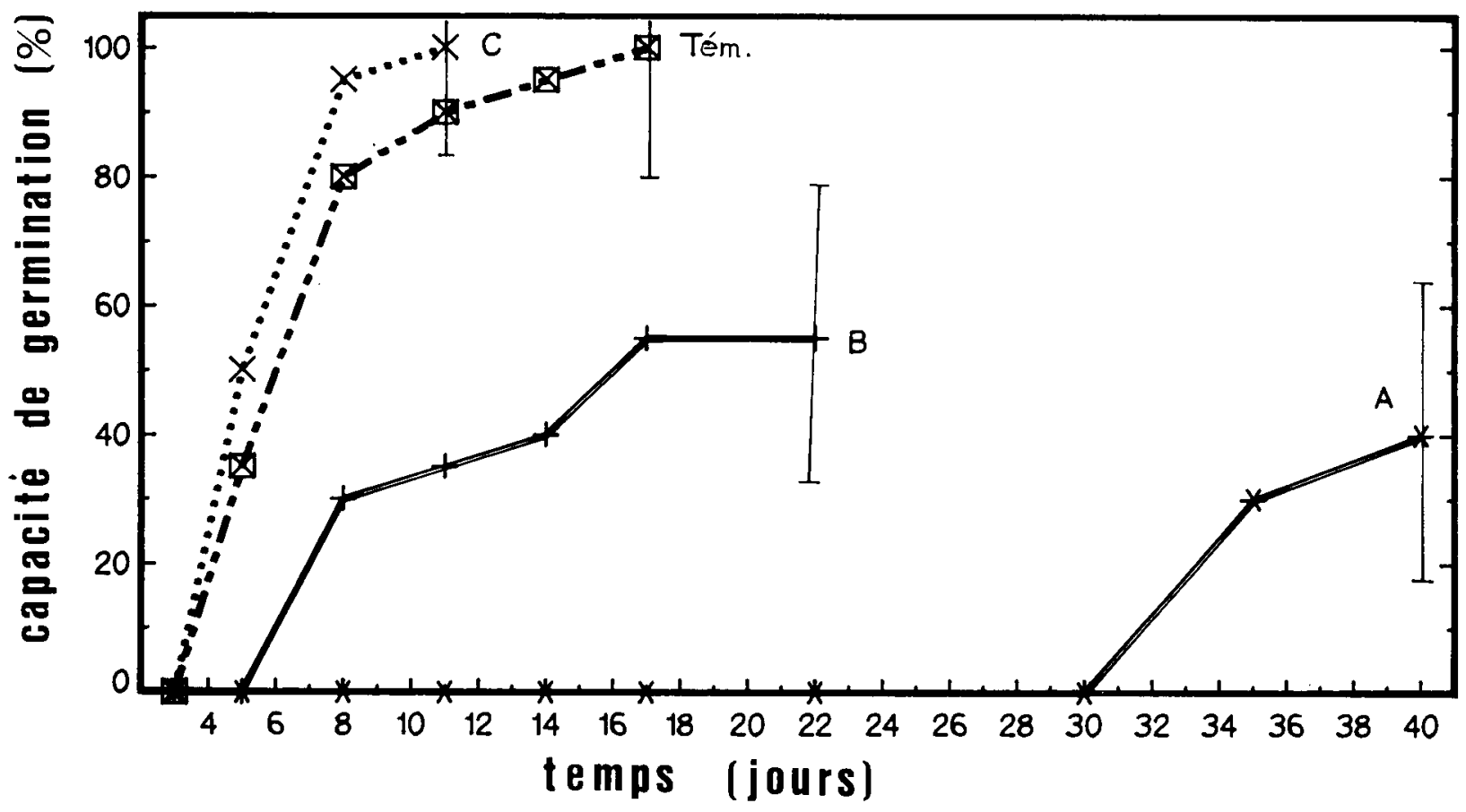

Fig 6. Germination d'embryons d'Heracleum sphondylium $\left(10^{\circ} \mathrm{C}\right.$, lumière) placés sur un milieu nutritif (milieu de Monnier enrichi de saccharose et de 3 acides aminés) : A. Issus de semences fraîches, atmosphère de $21 \%$ d'oxygène. B. Issus de semences fraîches, atmosphère de $15 \%$ d'oxygène. C. Issus de semences stratifiées 1 mois à $2{ }^{\circ} \mathrm{C}$ et $21 \%$ d' $\mathrm{O}_{2}$, atmosphère de $15 \%$ d'oxygène. Sont pris pour témoins (Tém) des embryons placés sur eau distillée et issus de semences stratifiées 2 mois à $2{ }^{\circ} \mathrm{C}$ et $21 \% \mathrm{O}_{2}$ (ceux issus de semences non stratifiées ne germant pas).

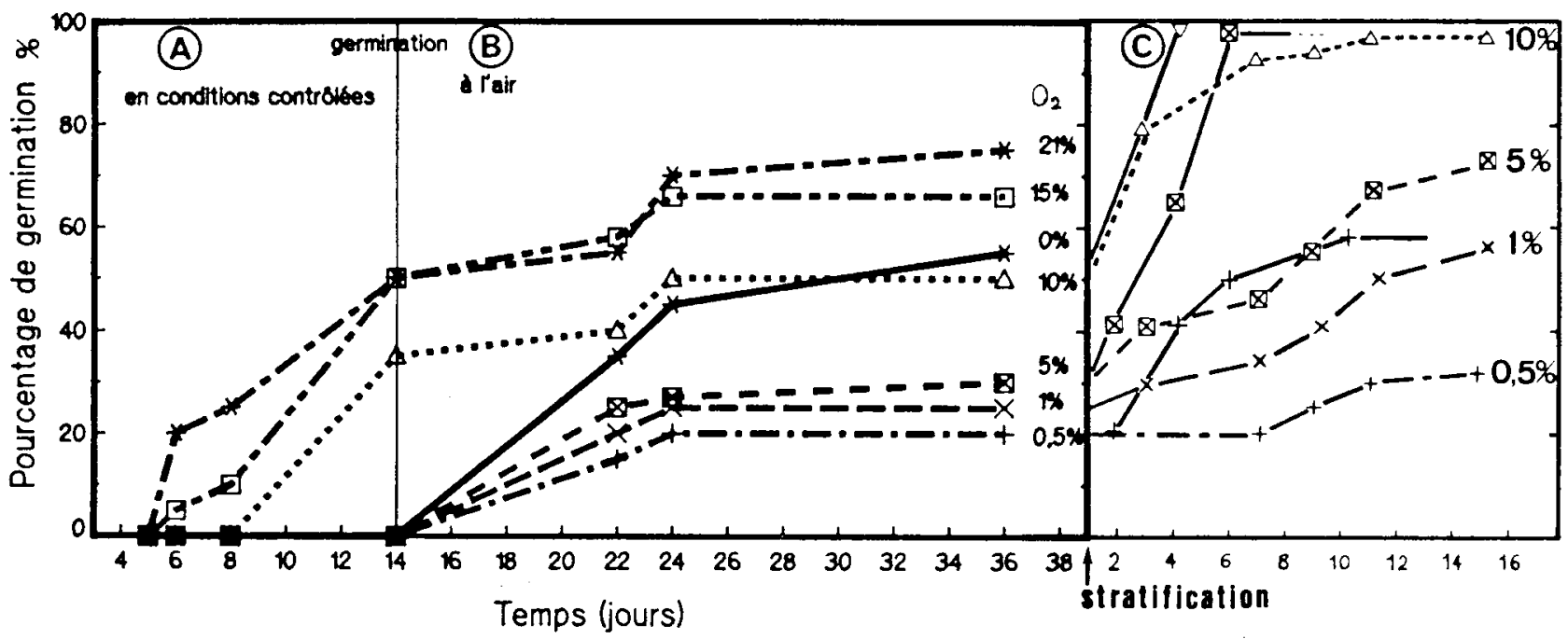

Fig 7. Influence de basses pressions d'oxygène sur la germination, à $15^{\circ} \mathrm{C}$ et à la lumière, d'akènes d'Heracleum sphondylium non dormants (prétraités 11 semaines à $2^{\circ} \mathrm{C}$ et $21 \%$ d' $\mathrm{O}_{2}$ ): A. Germination en conditions contrôlées d'oxygénation (les $\%$ d'O $\mathrm{O}_{2}$ correspondent à cette phase). B. Passage des semences restantes à $21 \%$ d'oxygène. C. Complément de germination après 4 (pointillés) ou 6 (en plein) semaines de séjour à $2{ }^{\circ} \mathrm{C}$ (germination à $10^{\circ} \mathrm{C}$, lumière, $21 \%$ d'oxygène) : n'ont pas été testées les semences ayant bien germé pendant la phase B $(0 \% ; 15 \% ; 21 \%)$ ainsi que la combinaison $1 \%-4$ semaines (problèmes sanitaires).

tion restent assez faibles : d'autant plus faibles que la teneur en oxygène était basse et d'autant plus faibles que le séjour en hypoxie était long (Mansour, 1990). II y a donc apparition d'une dormance secondaire. Un séjour au froid (fig 7C) permet de lever cette dormance secondaire.

Un séjour en anoxie donne, par contre, des résultats totalement différents (fig 7B) puisqu'après 
passage à l'air, de nombreuses semences germent correctement, montrant que l'anoxie ne permet pas l'installation d'une dormance secondaire. On observe cependant une germination décroissante quand l'anoxie se prolonge, mais sans amélioration par traitement au froid; des tests au tétrazolium ont montré que les embryons dégénèrent et meurent (Mansour, 1990).

Un séjour prolongé à la température de $30^{\circ} \mathrm{C}$ (fig 8), défavorable à la germination, permet aussi d'induire une dormance secondaire. Là aussi, une stratification permet de lever cette dormance. En fait, ce facteur agit sans doute en plaçant l'embryon en hypoxie, à la fois par une moindre disponibilité de l'oxygène dissous et par une intensification des oxydations au niveau des enveloppes.

\section{DISCUSSION}

\section{Caractéristiques de la germination}

\section{Nature de l'inaptitude à la germination}

Nous avons vérifié les résultats préliminaires de Stokes. L'immaturité embryonnaire de Heracleum peut être levée par une stratification à $2{ }^{\circ} \mathrm{C}$ au cours de laquelle la taille de l'embryon augmente (résultats non présentés, identiques à ceux de Stokes); 11 semaines à $2{ }^{\circ} \mathrm{C}$ sont nécessaires pour lever la dormance de nos semences, contre 14 semaines à $2{ }^{\circ} \mathrm{C}$ pour certaines semences plus dormantes de Stokes, alors que Lovett Doust et Lovett Doust (1982) obtiennent une capacité de $70 \%$ de germination après 3 mois à $5{ }^{\circ} \mathrm{C}$. La taille des embryons est multipliée par 6 environ dans nos expériences quand la dormance est levée; Stokes montre que cette croissance se poursuit régulièrement jusqu'à épuisement des réserves de l'albumen; la teneur en matière sèche augmente ainsi exponentiellement les 3 premiers mois au terme desquels elle est environ multipliée par 100.

Parmi les hypothèses d'explication de ce phénomène, Stokes élimine l'intervention d'un inhibiteur qui agirait à partir de $10^{\circ} \mathrm{C}$ et démontre qu'il ne s'agit que d'un problème de disponibilité des réserves; en effet, la durée de prétraitement nécessaire à $2{ }^{\circ} \mathrm{C}$ n'est aucunement modifiée par des séjours variables à $15^{\circ} \mathrm{C}$; elle est cumulative et fixe. Au-delà de $10^{\circ} \mathrm{C}$, l'embryon assimile les quelques réserves disponibles (jusqu'à augmenter de 2 fois sa longueur) puis cesse sa croissance faute d'approvisionnement; il ne la poursuit qu'après passage à basse température, condition nécessaire à une dégradation des réserves en éléments diffusibles.

Par la suite, Stokes, après analyse des transferts entre albumen et embryon à différentes températures, montre que les seuls éléments justifiant ces exigences thermiques sont les réserves protéiques; en analysant la dynamique de dégradation des granules de l'albumen, il suggère que la température agit sur l'émission d'enzymes protéolytiques par l'embryon, en-

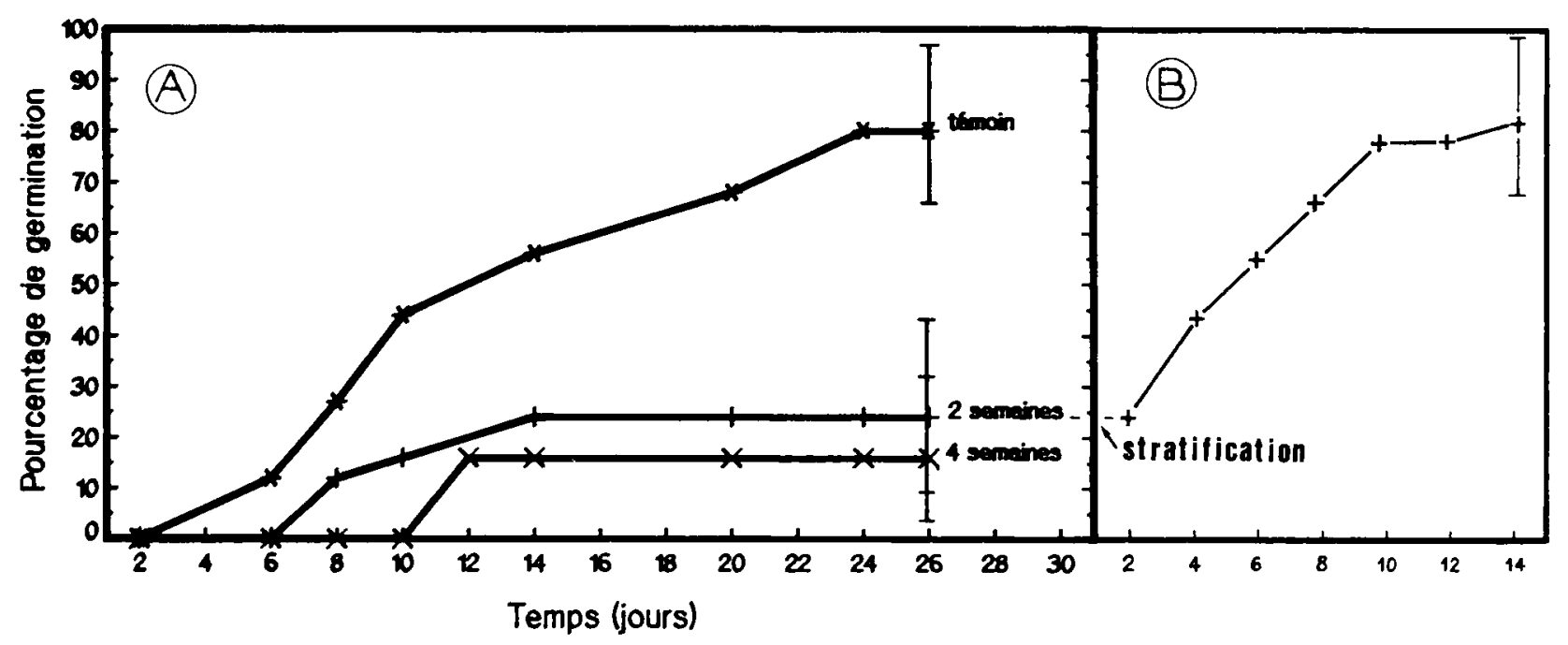

Fig 8. Influence d'un séjour de différentes durées ( 0 = témoin, 2 et 4 semaines) à température constante de $30^{\circ} \mathrm{C}$ sur la germination d'akènes d'Heracleum sphondylium $\left(10^{\circ} \mathrm{C}\right.$, lumière, $21 \%$ d'oxygène) : A. Germination à l'issue du prétraitement des akènes imbibés. B. Complément de germination après un séjour de 3 semaines à $2^{\circ} \mathrm{C}$ des akènes restants. 
zymes libérant certains acides aminés indispensables à une bonne croissance, comme l'arginine et la glycine. Nous avons pu fortement améliorer la germination d'embryons isolés par l'apport de ces acides aminés mais c'est la proline qui nous a fourni les meilleurs résultats.

L'absence de gonflement de l'embryon pendant les 3 premières semaines de stratification nous permet de proposer le processus suivant. Au cours des premières étapes du traitement s'effectue, sans évolution morphologique, une activation du métabolisme embryonnaire (germination sensu stricto) permettant la synthèse de certaines enzymes qui, après diffusion, déclenchent l'hydrolyse des réserves protéiques en éléments solubles; ces éléments diffusent en retour à travers une phase liquide jusqu'à l'embryon; la croissance démarre alors parallèlement à l'achèvement de la germination, les 2 phénomènes étant interdépendants.

Les cultures sur milieu nutritif ont montré que 4 semaines de froid suffisent amplement (fig 6); de même, des expériences de dessiccation d'akènes en cours de stratification ont montré une réversibilité de l'état physiologique pendant 5 semaines (Mansour, 1990); au-delà, on peut considérer que la germination est achevée et que la suite du traitement ne concerne que la croissance de la plantule.

\section{L'oxygénation}

Le comportement thermique d'Heracleum entre 0 et $30^{\circ} \mathrm{C}$ suggère une intéraction avec l'oxygène. Nous avons montré qu'aussi bien pendant la levée de dormance que pendant le développement d'une semence non dormante le besoin en oxygène était important. Les enveloppes placent en fait l'embryon en hypoxie et celui-ci n'aura ses besoins satisfaits ( $15 \%$ d'oxygène) qu'à une température basse; le facteur limitant pourrait donc être strictement la disponibilité de l'oxygène (Côme et Tissaoui, 1973).

La fixation au niveau des téguments semble être due aux substances phénoliques (activité polyphénoloxydasique) comme chez de nombreuses espèces (Côme, 1971; Jauzein, 1980; Lenoir et al, 1983 et 1986); il suffit de voir la teinte rapidement brune des papiers filtres après quelques semaines de stratification et la stimulation de la germination après changement de papier. Plus précisément, de nombreux auteurs ont analysé la composition en furannocoumarines des Heracleum, particulièrement abondantes dans les racines et dans les fruits, surtout au niveau des canaux sécréteurs (Jössang et al, 1969; Molho et al, 1971; Carbonnier-Jarreau et al, 1978; Carbonnier et Molho, 1978).

On a même noté la présence d'acétate d'octyle qui permet d'éviter un lessivage trop rapide des coumarines, et donc de maintenir plus longtemps leur action inhibitrice (Jössang et al, 1969). Enfin nous avons vérifié in vitro la sensibilité des semences d'Heracleum à ces substances puisque la germination d'akènes non dormants est totalement inhibée par une solution à $0,37.10^{-3}$ mol. I $^{-1}$ d'acide p-coumarique (Mansour, 1990).

La fixation au niveau de l'albumen pourrait être due au métabolisme lipidique, l'activité des lipoxygénases étant consommatrice d'oxygène (Lascorz, 1986). L'ensemble des enveloppes assure donc une régulation interne de la diffusion d'oxygène; l'embryon, d'abord placé en hypoxie dans les semences fraîches, va pouvoir disposer, au fur et à mesure de l'atténuation des inhibitions tégumentaires, d'une quantité d'oxygène suffisante, maintenue à une teneur optimale par la respiration des tissus de réserve.

Un séjour trop prolongé en hypoxie, en particulier si cette hypoxie est liée à une température élevée, provoque une entrée en dormance secondaire, phénomène observé sur de nombreuses espèces aussi bien au niveau des dormances embryonnaires (Côme et Tissaoui, 1968; Perino et Côme, 1977) que des inhibitions tégumentaires (Jauzein, 1979; Longchamp et Gora, 1979).

Comme chez le pommier, l'anoxie empêche l'installation de cette dormance, mais là s'arrête la comparaison car une anoxie est, par contre, incapable de lever la dormance primaire d'Heracleum; le fait que la semence d'Heracleum soit albuminée et que, parallèlement à la germination, interviennent des phénomènes de croissance n'est sans doute pas étranger à ce résultat négatif.

\section{Comportement en milieu naturel}

Les vérifications expérimentales du comportement d'Heracleum au champ faites par Mansour (1990) s'interprètent bien à la lumière des résultats obtenus in vitro.

En début de maturité morphologique, les dormances sont maximales. Des variations de cette dormance primaire s'observent en fonction du 
lieu de récolte (prédétermination variable) et de la date de la récolte. On peut mettre sur le compte de cette variabilité les écarts entre nos résultats et ceux de Stokes (1952a) dont les semences étaient un peu plus dormantes : luimême avait observé des différences selon les années de récoltes. De même, si l'on étudie l'intensité des inhibitions tégumentaires, les résultats sont beaucoup moins significatifs sur des récoltes tardives; on retrouve là l'influence du climat estival sur la postmaturation des semences mûres (Baskin et Baskin, 1979).

Quelle que soit l'intensité de la dormance primaire, l'immaturité embryonnaire masque toujours les inhibitions et est toujours suffisamment forte pour empêcher toute germination automnale. Chez Heracleum lehmannianum, la dormance est telle que 2 hivers successifs sont nécessaires avant que la germination soit possible (Carbonnier-Jarreau et al, 1978). L'humidité et le froid hivernal permettent à la fois une maturation progressive de l'embryon et une disparition des inhibitions tégumentaires. Dans le sol, la lixiviation des substances phénoliques hydrosolubles, constatée in vitro au cours d'une stratification, est complétée par l'action destructrice des microorganismes saprophytes; nous avons ainsi vérifié que sur des semences enfouies 5 mois dans le sol ces inhibitions avaient disparu. L'étude des semences enfouies montre que la dormance se lève au cours des mois de novembre, décembre et janvier. Ensuite, la germination pouvant s'effectuer à température basse, les premières phases de cette germination doivent se dérouler dès que l'oxygène, régulé par les activités des téguments et de l'albumen, devient suffisant au niveau de l'embryon.

En fin d'hiver, l'augmentation des températures est favorable à une croissance plus rapide; l'embryon n'est alors plus dépendant des températures basses. Les levées sont quasiment simultanées et apparaissent en mars-avril.

S'il y a eu travail du sol, l'indifférence des semences à la lumière leur permet de germer à toute profondeur. Nous avons vérifié qu'à $20 \mathrm{~cm}$ de profondeur, la dynamique des levées était identique à celle observée en surface aboutissant en avril à $95 \%$ de germination in situ. Cette inadaptation correspond à des espèces poussant sur sol stable; les mauvaises herbes adaptées aux champs labourés' acquièrent généralement une photosensibilité qui les empêche de germer en profondeur (Wesson et Wareing, 1969).

Heracleum est donc incapable de maintenir un stock semencier stable, ce qui explique sa sensi- bilité extrême au travail du sol. Cependant, une petite proportion $(5 \%$ dans notre expérimentation) ne germe pas; un tel comportement peut s'intensifier si la dormance primaire est encore trop forte en fin d'hiver, en particulier après succession d'un automne pluvieux et d'un hiver doux, et dans des sols engorgés où la diffusion de l'oxygène est très lente; or, en hypoxie, la levée de dormance ne s'effectue pas (fig 3 ), et si l'engorgement persiste, la germination ne peut s'effectuer (fig 2). Plus tard, les températures printanières et estivales intensifient la dormance; nous avons vérifié que, à l'image des résultats obtenus in vitro (figs 7 et 8 ), 2 semaines de stratification permettaient à des semences ayant persisté dans le sol de germer. Ces retardataires, par leur besoin en froid, ne germent qu'en mars-avril de l'année suivante; nous avons ainsi maintenu des semences parfaitement viables dans le sol pendant 22 mois.

Mais dans la nature, cette éventualité doit être négligeable car la viabilité ne peut être maintenue que sur semences enfouies dans le sol; les semences restant en surface sont sans doute tuées par la sécheresse estivale (Mansour, 1990). Or, les souches d'Heracleum ne supportent pas le travail du sol et ne persistent donc qu'en sol stable; les 2 situations sont exclusives.

\section{CONCLUSION}

Heracleum est donc un modèle intéressant chez lequel germination et croissance ne semblent pas, comme chez beaucoup d'espèces, séparées dans le temps. Mais des expériences beaucoup plus précises sont nécessaires pour dénouer les interactions étroites entre embryon et albumen, soit en précisant la localisation dans le temps et dans l'espace de l'action thermique (traitement au froid des embryons et des albumens séparés), soit en analysant les métabolismes enzymatiques et énergétiques dans les premières semaines de traitement au froid (métabolisme fermentaire éventuel de l'embryon). Alors seulement pourra-t-on déterminer s'il existe réellement une dormance germinative chez Heracleum.

Nous avons cependant répondu à notre souci d'interprétation du comportement d'Heracleum dans la nature. II est caractéristique de nombreuses espèces de régions tempérées à hiver bien marqué (Côme, 1982; Jauzein, 1980, 1989 et nombreuses observations), avec un optimum des capacités de germination situé à des tempé- 
ratures basses, et une action inhibitrice des températures supérieures à $20^{\circ} \mathrm{C}$, responsables de l'induction de dormances secondaires; un besoin relativement important en oxygène suffit à l'expliquer.

Cette espèce illustre très bien l'inadaptation des phénomènes de dormance embryonnaire à des perturbations aléatoires du milieu. De telles dormances induisent une dynamique homogène et prévisible des levées au champ. Seules les inhibitions des enveloppes semblent capables d'assurer une réelle hétérogénéité des semences, le plus sûr garant de la survie des annuelles.

\section{REFERENCES}

Baskin JM, Baskin CM (1979) Studies on the autoecology and population biology of the weedy monocarpic perennial, Pastinaca sativa. J Ecol 67, 601-610

Carbonnier J, Molho D (1978) Variations selon l'organe du contenu coumarimique de quelques Ombellifères. Bull Mus Natl Hist Nat Paris, $3^{\mathrm{e}}$ ser, 522, 17-21

Carbonnier-Jarreau MC, Carbonnier J, Molho D (1978) Variations saisonnières des quantités totales de coumarines de la racine d'Heracleum lehmannianum Bge (Umbelliferae). Bull Mus Natl Hist Nat, Paris, $3^{e}$ sér, 522, 13-16

Come D (1971) Dégazage des enveloppes séminales lors de leur imbibition. Physiol Vég 9, 447-452

Come D (1982) Facteurs généraux de la germination. In: Croissance et développement (Mazliak $\mathrm{P}$, ed) Hermann, Paris, 159-184

Come D, Tissaoui T (1968) Induction d'une dormance embryonnaire secondaire chez le Pommier (Pirus malus L) par des atmosphères très appauvries en oxygène. CR Acad Sci Paris, 266, sér D, 477-479

Come D, Tissaoui T (1973) Interrelated effect of imbibition, temperature and oxygen in seed germination. In: Seed ecology (Heydecker W, ed) Butterworths, Londres, 157-168

Flemion F, Henrickson ET (1949) Further studies on the occurrence of embryoless seeds and immature embryos in the Umbelliferae. CR Boyce Thompson Inst 15, 291-297

Janiesch P (1971) Zur Physiologie der Nachreife von Umbelliferen nitrophiler Säume. Flora 160, 518-525

Jauzein $P$ (1979) Physiologie de la germination des graines de Sinapis arvensis $L$. Thèse doct $3^{e}$ cycle, Paris, $161 \mathrm{p}$

Jauzein $P$ (1980) Caractéristiques physiologiques de la germination de Sinapis arvensis $\mathrm{L}$. Vie Coll Int Ecol Biol Syst des mauvaises herbes 1, 63-72

Jauzein P (1986) Échelonnement et périodicité des levées de mauvaises herbes. Bull Soc Bot Fr 133, Lettres Bot, 155-166
Jössang P, Carbonnier J, Molho D (1969) Étude des constituants de l'Heracleum mantegazzianum cultivé à la Jaysinia. Trav Lab La Jaysinia 3, 187-195

Kobilinsky A, Decoux G, Derrien A (1988) Modli, programme d'analyse de variance et covariance. INRA, laboratoire de Biométrie, Versailles

Lascorz M (1986) Recherches sur l'influence de quelques enzymes d'oxydoréduction des enveloppes sur la dormance des semences d'Avoine (Avena sativa $\mathrm{L}$ ). Thèse doct $3^{e}$ cycle, Paris, $115 p$

Lenoir C, Corbineau F, Come D (1983) Rôle des glumelles dans la dormance des semences d'Orge. Physiol Vég 21 (4), 633-643

Lenoir C, Corbineau F, Come D (1986) Barley (Hordeum vulgare) seed dormancy as related to glumella characteristics. Physiol Plant 68, 301-307

Longchamp JP, Gora M (1979) Influence d'anoxies partielles sur la germination de semences de mauvaises herbes. Oecol Plant 14, 121-128

Lovett-Doust J, Lovett-Doust L (1982) Life history patterns in British Umbelliferae: a review. Bot $J$ Linn Soc 85, 179-194

Mansour A (1990) Écophysiologie comparée de la germination de quelques espèces de la famille des Umbelliferae : Aethusa cynapium L, Heracleum sphondylium L, Pastinaca sativa $\mathrm{L}$ et Tordylium maximum $L$, Thèse doct Rennes, $133 p$

Molho D, Jössang P, Jarreau MC, Carbonnier J (1971) Dérivés furannocoumariques du genre Heracleum et plus spécialement de Heracleum sprengelianum Wight et Arn et Heracleum ceylanicum Gardn ex Clarke. Étude phylogénique. In: The biology and chemistry of the Umbelliferae. Bot J Linn Soc sup 1, 64, 337-360

Monnier M (1971) Action des conditions de stérilisation sur la valeur nutritive des milieux utilisés pour la culture des embryons isolés de Capsella bursapastoris. Rev Gén Bot 78, 57-60

Perino C, Come D (1977) Influence de la température sur les phases de la germination de l'embryon de Pommier (Pirus malus L). Physiol Vég 15, 469-474

Roberts HA (1979) Periodicity of seedling emergence and seed survival in some Umbelliferae. J Appl Ecol 16, 195-201

Stokes P (1952a) A physiological study of embryo development in Heracleum sphondylium L. I. The effect of temperature on embryo development. Ann Bot NS 16, 439-447

Stokes P (1952b) II. The effect of temperature on after-ripening. Ann Bot NS 16, 571-576

Stokes $P$ (1953a) III. The effect of temperature on metabolism. Ann Bot NS 17, 157-173

Stokes P (1953b) The stimulation of growth by low temperature in embryos of Heracleum sphodylium L. J Exp Bot 4 (11), 222-234

Wesson G, Wareing PF (1969) The induction of light sensitivity in weed seeds by burial. $J$ Exp Bot 20 , 414-425 